\title{
DISTANCE-REGULAR ANTIPODAL COVERING GRAPHS
}

\section{R.E.L. ALDRED}

This thesis sets out to investigate the highly combinatorially regular class of graphs known as distance-regular graphs. In particular attention is focussed on the special class of distance-regular antipodal covering graphs.

Following the introduction, the thesis develops the general theory of distanceregular graphs, drawing motivation, at times, from the theory of distance-transitive graphs. The combinatorial regularity requirements of a given graph are detailed by means of an intersection array and the question of whether or not there exists a graphical realisation of a given intersection array is considered. Although this question remains unanswered, some results obtained in answer to related questions are presented. In particular it is determined that there are only finitely many distance-regular graphs with a pair of consecutive equal subdegrees. A classification scheme is developed to enable a logical division of the class of grpahs considered. The scheme developed analogous to the Smith classification scheme for distance-transitive graphs. This result is not new, however the proof presented here is new and succinct.

In the third chapter the class of distance-regular graphs under consideration is further restricted. Unlike the classification scheme which was developed in the second chapter, the concern here is not with simply classifying the distance-regular graphs by the properties of the graphs but also with finding the relationship to other known distance-regular graphs. Drawing from the theory of Permutation Groups and Topology a "quotient" of a distance-regular graph is defined and refined until eventually the definition of $n$-fold distance-regular antipodal covering graphs is reached. During the course of this discussion several new perspectivews of covering graphs are presented and the relationships between the covering graphs and the graphs that they cover are investigated. A correspondence is established between the eigenvalues and their multiplicities in a distance-regular graph and its antipodal covering graph.

The problem of which graphs admit $n$-fold distance-regular antipodal covering graphs provides the main motivation for the thesis. The known results on maximum

Received 18 July 1988.

Thesis submitted to the University of Melbourne, December 1986. Degree approved, March 1988. Supervisor: Professor C.J. Thompson.

Copyright Clearance Centre, Inc. Serial-fee code: 0004-9729/89 \$A2.00+0.00. 
covers in general, and submaximum covers for complete graphs, are presented in survey fashion. In addition, more information on $n$-fold antipodal coverings of complete graplis is provided. In the closing stages of Chapter Three the possible existence of $n$-fold distance-regular antipodal covers of the incidence graphs of symmetric designs is investigated. This investigation establishes a link between the covers of symmetric designs and the covers of complete graphs.

Chapter Four addresses the question of which $n$-fold distance-regular antipodal covers of complete bipartite graphs exist. In the first part of the chapter a survey is given of the pre-existing results in the area and new proof is provided of the equivalence of 2-fold distance regular antipodal covers of complete bipartitie graphs to Hadamard matrices.

The main result of the thesis is a characterisation of the $n$-fold covers of complete bipartite graphs. It shows the equivalence of an $n$-fold distance-regular antipodal covering of a compelte bipartite graph to a system of resolvable designs. In the discussion following the theorem the previously determined characterisations of particular cases are extracted from the general characterisation. In so doing several links between known combinatorial structures are indicated.

To round off the chapter, constructions of several families of coverings are presented and discussed. Included is a construction of $n$-fold coverings of $K_{n^{2}, n^{2}}$ from affine planes of order $n$ when $n$ is prime. This construction is of interest not only because of the fact that it uses the characterisation developed earlier, but also because it makes use of the classical combinatorial structure of affine planes. Another construction uses a 1-factorisation of the complete graph on six vertices to produce a 3-fold cover of $K_{6,6}$.

A final section is devoted to directions of possible future research in related areas.

\author{
Department of Mathematics and Statistics \\ University of Otago \\ P.O. Box 56 \\ Dunedin \\ New Zealand
}

